\title{
May ultrasound probe size influence pain perception of needle piercing during transrectal prostate biopsy? A prospective evaluation
}

\author{
Andrea Fabiani ${ }^{1}$, Lucilla Servi $^{1}$, Alessandra Filosa ${ }^{2}$, Fabrizio Fioretti ${ }^{1}$, Valentina Maurelli ${ }^{1}$, \\ Flavia Tombolini ${ }^{3}$, Matteo Tallè ${ }^{3}$, Gabriele Mammana ${ }^{1}$ \\ ${ }^{1}$ Surgery Department, Section of Urology, ASUR Marche Area Vasta 3, Macerata Hospital, Macerata, Italy; \\ ${ }^{2}$ Section of Pathological Anatomy, Department of Clinical Pathology, ASUR Marche Area Vasta 3, Macerata Hospital, Macerata, Italy; \\ ${ }^{3}$ Urologic Clinic, Polytechnic University of Marche Region, Italy.
}

\section{Summary Introduction and Objective: Transrectal ultrasound guided prostate biopsy}

(TRUS-BX) is the definitive step in the diagnosis of prostate cancer (CaP). Patients (pts) generally experience significant pain during the procedure at the point that biopsy should be accompanied by some form of anesthesia. Several different factors influence pain perception (PP) during TRUS-Bx. In our study we want to assess that the use of an ergonomic smaller sized probe reduces PP during the procedure independently from the administration of local anesthesia or pain relieving drugs.

Materials and Methods: This was a prospective, randomized study in which 114 pts who underwent TRUS-Bx due to abnormal PSA and/or to digital rectal examination (DRE) suspicious findings were considered eligible. Pts were split in two TRUS-Bx groups into which we used two different sized ultrasound probes. In group 1, 61 pts underwent TRUS-Bx with ALOKA end fire probe (size $74 \mathrm{~mm}$ ). In group 2, 53 pts underwent TRUS-Bx with B-K Type 8818 probe (size 58 $\mathrm{mm}$ ). Both groups were treated with no local anesthesia or pain relieving drugs. Pain was evaluated three times using a 10-point visual analogue scale (VAS), during the DRE (VAS 1), during the insertion of the probe (VAS 2) and during the needle piercing (VAS 3).

Results: Mean age of pts was 68.03 (SD 8.51); mean tPSA and mean prostate volume was 7.75 (SD 4.83) and 45.17cc (SD 17.7), respectively. The two groups were homogeneous respect to tPSA $(p=0.675)$ and to prostate volume $(p=$ $0.296)$; age was significantly different $(p=0.04)$ between Group 1 (65.93) and Group 2 (70.43), whereas no statistically significant correlation between VAS 3 and age was observed ( $p=0.179)$. Analyzing pain perception, we found no statistically significant difference between the two groups in DRE (VAS 1; $p=0.839$ ); on the contrary, patients in Group 1 experienced on average more pain than other in Group 2 both during the insertion of the probe (VAS 23.49 vs 1.09; $p<0.001)$ and during the needle piercing VAS 3 (2.8 vs 2.00; $p<0.05)$. The discomfort during probe insertion and manipulation was perceived as very high (VAS $2>$ 5) in $42.6 \%$ of patients in Group 1 and in 9.4\% in Group 2. Globally, the procedure was well tolerated (mean VAS score $<3$ ) in $77 \%$ of patients in Group 1 and in 90\% in Group 2. The proportion of patients who experienced more than moderate pain (VAS > 5) during needle piercing ranged $24.6 \%$ in Group 1 to $18.9 \%$ in Group 2.
Conclusions: Patients who underwent a TRUS-Bx with the 58-mm circumference probe were found to experience lower degree of pain not only during the insertion of the probe through the anal sphincter, but also in the moment of needle piercing.

KEY WORDS: Transrectal ultrasound; Prostate biopsy; End fire probe; Pain; Size.

Submitted 1 November 2015; Accepted 4 December 2015

\section{INTRODUCTION}

Prostate biopsy is one of the most common procedures performed in the urologist's office today. Despite developments in the field of prostate imaging recently reached, it is still considered the standard procedure for diagnosing prostate cancer (1-2). During the years, the minimization of the sampling error has been the goal of urologic community. However, the increase in the number and location of cores, if on the one hand it led to an improvement of prostate cancer detection rate, on the other led to an increase of patient discomfort. Patients may generally experience a significant pain during the procedure at the point that biopsy should be accompanied by some form of anesthesia (3). Conversely, improvements in anesthesia techniques have allowed physicians to sample with a great number of cores and at different locations in the gland, achieving patient compliance and, finally, being able to perform the procedure in an office setting. However, despite these considerations, the use of anesthesia is still under debate because of doubt of its real benefits and the associated costs (4). Two factors are usually responsible for pain during transrectal prostate biopsy: anal pain due to ultrasound probe, that causes pressure and stretching of muscle fibers, and pain at insertion of the needle through the prostate (5). Are these factors related? May ultrasound probe geometry influence pain perception of needle piercing during transrectal prostate biopsy? To contribute in the answers of these questions, we present a prospective randomized 
evaluation assessing the role of the use of an ergonomic smaller sized probe on reducing pain perception during ultrasound guided prostate biopsy, independently from the administration of local anesthesia or pain relieving drugs.

\section{Material AND MEthods}

In this prospective randomized study, 114 patients underwent TRUS guided prostate biopsy. They had no history of previous prostate biopsy, chronic prostatic pain or pelvic pain syndrome, anal surgery, concomitant analgesic medication or any other medical condition that
Additionally, we determined the relationship between the level of pain, prostate volume, age and PSA. Statistical evaluation was performed Student test T by Med Calc System. P value less than 0.05 was considered statistically significant.

\section{RESULTS}

The study groups were comparable in PSA and prostate size. Age was significantly high in Group 2. (Table 1). The mean pain scores in Group 1 during DRE (VAS1), probe insertion and ultrasound procedure (VAS2) and during biopsy (VAS3) were $0.42 \pm 0.66,3.49 \pm 3.17$ and

\section{Table 1.}

Patients characteristics.

\begin{tabular}{|c|c|c|c|c|}
\hline Patients characteristics & Total $(n=114)$ & Group $1(n=61)$ & Group $2(n=53)$ & p-value \\
\hline Mean age (yr) & $68.03 \pm 8.51$ (range $50-85$ ) & $65.93 \pm 7.54$ (range $51-81)$ & $70.43 \pm 8.98$ (range $50-85$ ) & 0.04 \\
\hline$\overline{\text { Mean prostate size }(\mathrm{ml})}$ & $45.17 \pm 17.70$ (range 20-120) & $46.79 \pm 19.86$ (range 20-120) & $43.30 \pm 14.79$ (range $20-78 \mathrm{ml})$ & 0.296 \\
\hline$\overline{\text { Mean PSA (ng/ml) }}$ & $7.75 \pm 4.83$ (range $0.66-31$ ) & $7.93 \pm 4.69$ (range 0.66-24.81) & $7.55 \pm 5.03$ (range $0.82-31$ ) & 0.675 \\
\hline
\end{tabular}

could potentially interfere with pain assessment. Indications to prostate biopsy were an abnormal PSA and/or a suspicious findings on digital rectal examination (DRE). Patients was randomized into two Groups in which we used two different sized ultrasound probes. In Group $1(n=61)$ patients underwent TRUS biopsy using an ALOKA machine with a 5-12 MHz multi-frequency convex probe "end-fire" sized $74 \mathrm{~mm}$. In Group 2 ( $\mathrm{n}=$ 53) patients underwent TRUS biopsy with BK MEDICAL machine equipped with an end fire probe (Type 8818) sized $58 \mathrm{~mm}$. As explained to any patient in informed consensus, both groups were treated with no local anesthesia or pain relieving drugs. Only an aqueous gel was used to perform DRE and probe insertion. The procedures were performed by the same operator on the patients in the left lateral decubitus ("Sims position") after emptying of the bladder, according to believed that the state of bladder repletion may be an element of discomfort during the prostate mapping biopsy performance (6). Antibiotic prophylaxis was given to the patients (oral fluoroquinolone $1-2 \mathrm{~h}$ before the procedure and three days after). Twelve random cores biopsies were obtained, 6 from each lobe of the prostate gland, according to Gore scheme (7). After transrectal ultrasound performance, conducted assessing the prostatic diameter, the volume of the whole prostate, the transition zone, capsular and seminal vesicle characteristics, as well as morphological description of potential pathological features (8), sampling was carried out with a 18-Gauge Tru-Cut needle powered by an automatic spring-loaded biopsy disposable gun. Pain was evaluated after procedure asking the patient to sign in a 10-point visual analogue scale (VAS) the pain degree felt during each phase of procedure. In VAS scale, number 0 represented absence of pain and number 10 the maximum pain perceived in life. The VAS evaluation was differentiated in three scales considering the pain perceived during the DRE (VAS 1), during the insertion of the probe and the movements associated (VAS 2), and during the needle piercing (VAS 3).
Table 2.

Results for mean pain scores.

\begin{tabular}{|lccc|}
\hline & Group 1 & Group 2 & p value \\
\hline $\begin{array}{l}\text { Mean pain score during EDR (VAS 1) } \\
\text { manipulation (VAS 2) }\end{array}$ & $0.42 \pm 0.66$ & $0.45 \pm 0.72$ & 0.839 \\
\hline Mean pain score during BIOPSY (VAS 3) & $2.8 \pm 2.22$ & $2.0 \pm 2.03$ & 0.05 \\
\hline
\end{tabular}

$2.8 \pm 2.22$ respectively. The same mean pain scores in Group 2 were $0.45 \pm 0.72,1.09 \pm 1.68$ and $2 \pm 2.03$ respectively (Table 2). While age had shown a significant difference ( $p=0.04)$ between Group 1 (65.93) and Group 2 (70.43), no statistically significant correlation

\section{Figure 1.}

Correlation between patient age and pain perceived during probe insertion.

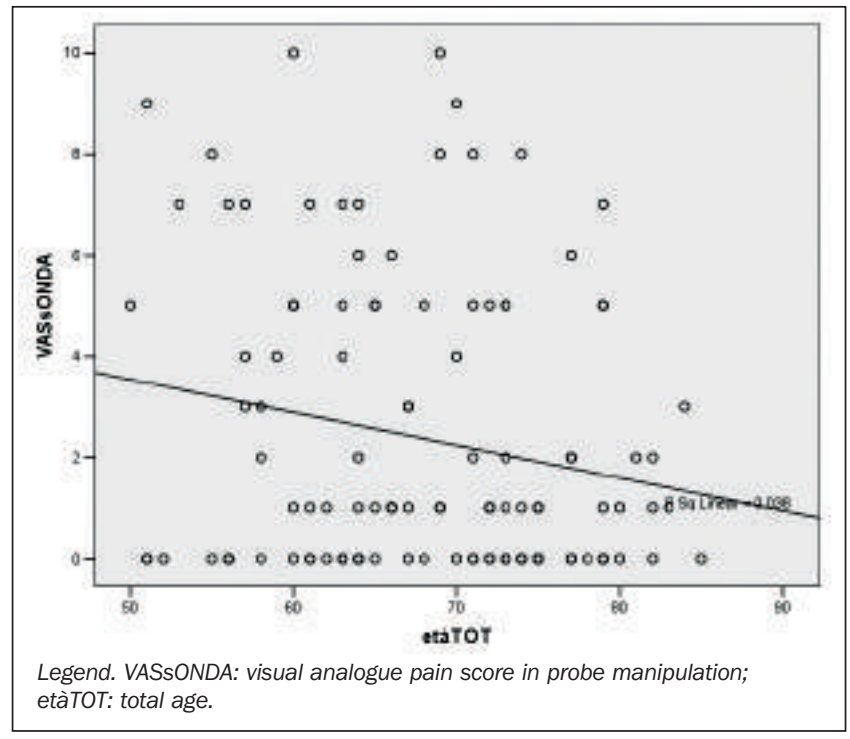


Figure 2.

Correlation between patient age and pain perceived during needle piercing.

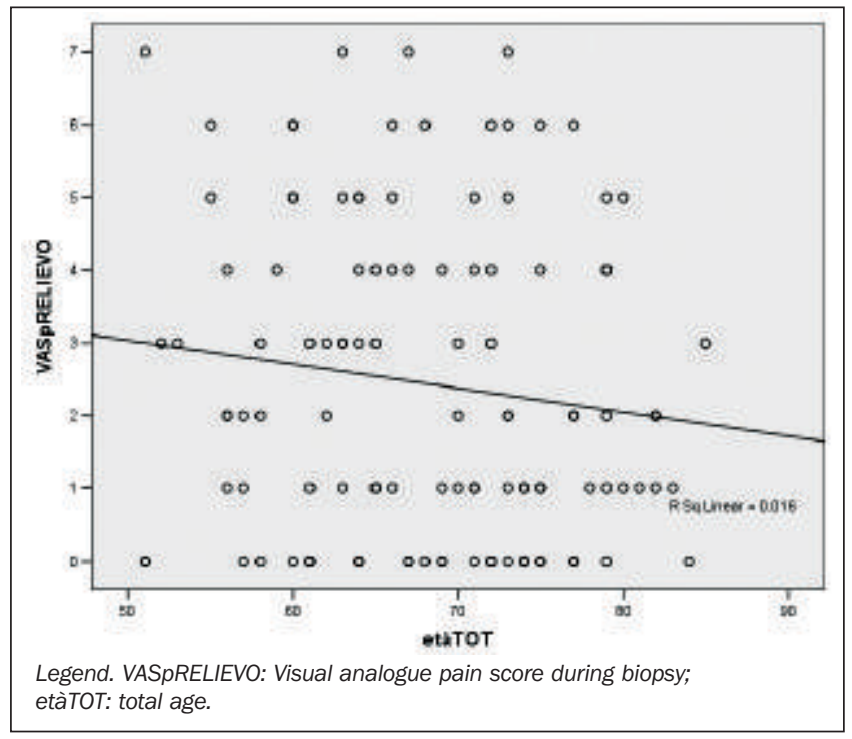

Figure 3.

Correlation beetween patient age and pain perceived during digital rectal examination.

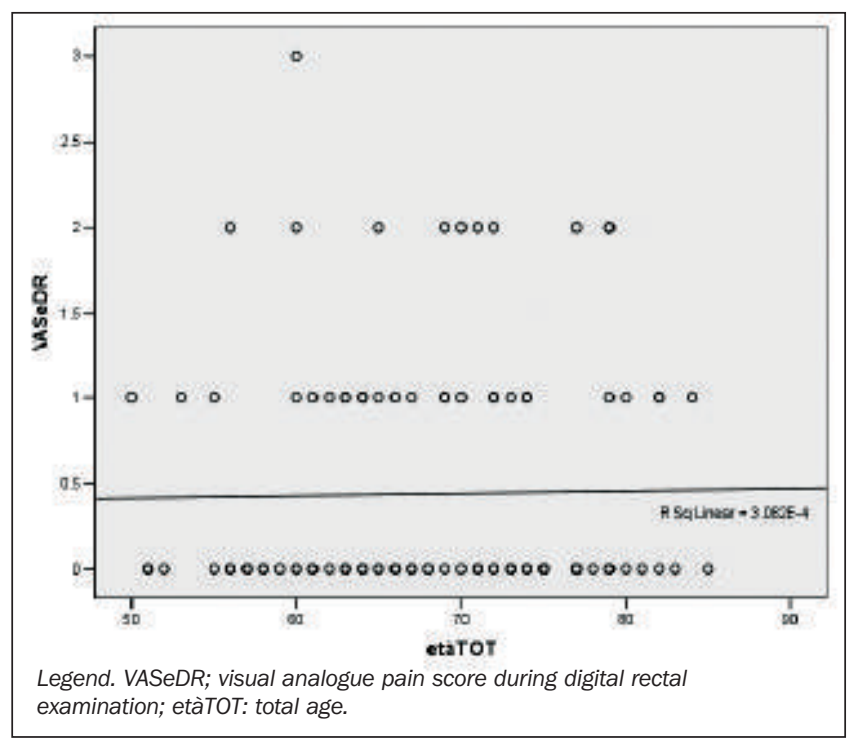

between VAS 3 and age was observed $(p=0.179)$ (Figures 1-3). Globally, the procedure was well tolerated (mean VAS score $<3$ ) in $77 \%$ of patients in Group 1 and in $90 \%$ in Group 2. Probe insertion or manipulation during the procedures (VAS2) were felt by patients as more than a moderate pain (VAS > 5) in $42.6 \%$ in Group 1 and $9.4 \%$ in Group 2. The pain perception during needle piercing (VAS 3) was high (VAS > 5) for 15 patients in Group 1 (24.6\%) and 10 patients in Group 2 (18.9\%). There were only minor complications and were managed on an outpatient basis without admission. Both groups were comparable in terms of complications. There was no significant difference in the cancer detection rate (65.6\% in group $1 ; 66 \%$ in group 2 ).

\section{Discussion}

It is the experience of every urologist that most patients experience moderate to severe pain during the prostate biopsy procedure (9) and their discomfort appears to be proportional to the number of cores taken (10-11). There is now a strong evidence in the current literature that anesthesia and/or analgesia improves patient tolerance and comfort (5). Therefore, it is suggested that all urologists should introduce it in clinical practice as a routine part of the procedure whatever the patient characteristics and biopsy scheme (12-13). Of the various methods of peri-prostatic nerve block alone or associated with lidocaine gel has been shown to be safe, easy to perform and highly effective. With limitations, it can be considered the gold standard at the moment even if the optimal technique remains to be established (14-15). However, several different factors contribute to discomfort and pain during ultrasound guided transrectal biopsy. Omitting the psychological patients stress attributable to fear of the potential diagnosis of cancer, to the anal route of penetration and to the fact that the examined organ is part of the male sexual system, it is recognized that the introduction and movement of TRUS probe into the rectum, the geometry of the ultrasound probe itself, the needle piercing through the rectal wall, the needle passage through the prostate capsule and the number of biopsy cores taken are the most determinant etiologic factors of pain during the procedure (5). As suggested by Giannarini et al. (16) the variability of perceived discomfort might be related to differences in anorectal compliance. They suggested the possibility of omitting anesthesia in those patients with high anorectal compliance. Others (2) have stated that pressing the probe against the rectum might minimize the discomfort of the biopsy needle traversing the rectal mucosa. We think that anorectal tone is the most determinant factor of perceived pain during ultrasound guided transrectal prostate biopsy. Similarly to what happened with the transition from rigid to flexible cystoscopy, the use of an ergonomic probe may greatly may reduce the patient discomfort during the procedure. As suggested by Moussa et al. (17) it must be focalized into the role of transrectal probe configuration, the probe design and needle guide in determining pain during the ultrasound guided prostate biopsies. They analysed prospective data on 1114 patients undergoing the procedure in a three years period. From results derived from analysis of patient's responses of a 10-point visual analogue pain scale related to the consecutive steps of prostate biopsy (probe insertion, application of peri- prostatic nerve block and the obtaining of prostate biopsies cores), they concluded that probe configuration may affect pain during each step of TRUS-guided prostate biopsy. Rochester et al. (18), in his double-blind randomized controlled trial of topical glyceryl trinitrate use during ultrasound guided transrectal prostate biopsy, concluded that although decreasing anal sphincter tone is effective for decreasing pain associated with biopsy, its role may be in combination with local anesthetic infiltration, which together address two different aspects of pain associated with biopsy. In the other hand, the use of anesthesia in performing prostate biopsy is still under debate because of doubt of its real benefits and the associated costs (4). Moreover, peri- prostatic 
nerve block causes a lengthening of the procedure and requires the patient to undergo additional injections. These considerations led us to the idea to investigate if the reduction of the mechanical stretching of sensory fibers innervating the part of the anal canal distal to the dentate line using an ergonomic probe might cause a discomfort reduction to the patient not only during the introduction of the probe itself but also in pain perception of needle piercing. Few studies have accessed the influence of size probe on pain perception during TRUS guided prostate biopsy. Koprulu et al. (19) asserted that under adequate local anesthesia there is no difference in pain perception between a larger or a smaller probe. They evaluated one hundred and seventy patients who underwent TRUS guided biopsies, divided into three TRUS biopsies groups. Group I (60 pts) underwent TRUS biopsy with newer BK Type 8808 probe (circumference $58 \mathrm{~mm}$ ) under injectable periprostatic anesthesia, Group II (60 pts) underwent TRUS biopsy with BK Type 8551 probe (circumference $74 \mathrm{~mm}$ ) under injectable periprostatic anesthesia, and Group III (50 pts) underwent TRUS biopsy with BK Type 8551 probe (circumference $74 \mathrm{~mm}$ ) without local anesthesia. Peri-prostatic injection anesthesia was performed with $10 \mathrm{cc}, 1 \%$ lidocaine (5 cc on each side) $10 \mathrm{~min}$ before procedure. Pain was assessed using a 10-point modified visual analog scale (VAS) 15 min after the biopsy procedure. Most of the patients experienced no pain to slight pain in Groups I and II, but $66 \%$ of the patients had more than moderate pain (VAS $\geq 5$ ) in Group III with mean VAS score statistically higher than the other two groups (Group I vs. III, P = 0.0001; Group II vs. III, $\mathrm{P}=0.0001)$. Mean VAS score was not statistically different between Group I and II $(\mathrm{P}=0.126)$. No statistically significant difference in VAS pain perception was observed between different age categories within the Group I, II, and III. Authors concluded that in the absence of injectable local anesthesia, larger probe (74 $\mathrm{mm}$ ) results in much higher VAS pain perception than same size and smaller $(58 \mathrm{~mm})$ probe used under injectable local anesthesia. However, under injectable local anesthesia, the size of the transrectal probe $(58 \mathrm{~mm}$ vs. $74 \mathrm{~mm}$ ) does not result in any different pain perception during TRUS biopsy. In our part, we investigated how the ergonomic smaller sized probe can reduce pain perception, making the procedure more comfortable and well tolerated by the patients, without performing any form of anesthesia. We built the randomization of the study in order to eliminate some possible confounders. The patients in both group underwent the same number of needle piercing (twelve) avoiding to perform a periprostatic nerve block. The pain perceived was the result of digital rectal examination (during which the same operator provide to insert a lubricant gel), the probe insertion and/or movements and the prostatic tissue biopsy. The two groups were similar in terms of clinical data (PSA, prostate volume, DRE). We noted a difference in terms of age. Group 2 (smaller sized probe) was characterized by an older age than Group 1. Some literature data demonstrated age $>65$ years as "a natural anesthetic" in pain perception during the transrectal ultrasound-guided prostate biopsy procedure (20). In our data, mean age was > 65 years in both group and statistical analysis revealed no significant correlation between VAS 3 (pain perceived during needle piercing) and age $(\mathrm{p}=0.179)$ (Figure 3). The only significant different variable between the two groups was the size of the probe used. As we expected, using a $58 \mathrm{~mm}$ probe instead of $74 \mathrm{~mm}$ reduced very significantly pain perception during the insertion of the probe (VAS 2). Moreover pain perception is reduced not only during the insertion of the probe, but also during needle piercing biopsy (VAS 3), always significantly. In effect, there are several data in scientific literature supporting the evidence that probe insertion is more painful than prostatic biopsy performed under peri-prostatic nerve block. For example, Philip et al. (21), evaluating in a prospective manner the efficacy of bi-apical vs bibasal periprostatic nerve block during 12 core ultrasound guided prostate biopsy, found that patients who experienced greater pain with the introduction of the probe also reported more pain with the biopsy procedure. These data may suggest that most of the pain the patients experienced during TRUS guided prostate biopsy is not just related to the piercing itself, but the insertion of the probe has some key role on overall pain, probably due to the tone of anal sphincter. If the only ergonomic geometry of the probe in a compliant anus reduces also the perceived pain during cores sampling, then it is not so necessary a peri-prostatic nerve block but a maneuver that locally augment anal compliance with the opportunity to select the cases in which is necessary a form of analgesia, just during anal tone assessment with digito-rectal examination as suggested by Onur et al. (22). We need further studies to evaluate that.

\section{Conclusion}

Performing a TRUS guided prostate biopsy with the $58 \mathrm{~mm}$ circumference probe patients were found to experience lower degreesof pain not only during the insertion of the probe trough the anal sphincter, but also at the moment of needle piercing. Ultrasound probe geometry may influence pain perception during the prostate biopsy procedure and may be determinant in selecting patients in which it is needed some form of analgesia.

\section{AUthors' CONTRIBUtions}

All Authors participated in the design and conduct of the study. All Authors reviewed and approved the final version of the manuscript.

\section{References}

1. Barentsz JO, Richenberg J, Clements R, et al. ESUR prostate MR guidelines 2012. Eur Radiol. 2012; 22:746-757.

2. Ramey CR, Halpern EJ, Gomella LJ. Ultrasonography and biopsy of the prostate: prostate biopsy techniques and outcomes; in Wein AJ, Kavoussi LR, Novick AC, Partin AW, Peters CA (eds): CampbellWalsh Urology, ed 9. Philadelphia, Saunders. 2007, pp 2887-2892.

3. Luscombe CJ, Cooke PW. Pain during prostate biopsy. Lancet. 2004; 363:1840-1841.

4. Shinohara K, Master VA, Chi T, et al. Prostate needle biopsy techniques and interpretation; in Vogelzang NJ, Scardino PT, Shipley 
WU (eds): Genitourinary Oncology. Philadelphia,Lippincott, Williams \& Wilkins, 2006, pp 111-119.

5. Maccagnano C, Scattoni V, Roscigno M, et al. Anaesthesia in transrectal prostate biopsy: which is the most effective technique. Urol Int. 2011; 87:1-13.

6. Dell'Atti L. Lidocaine spray administration in transrectal ultrasound guided prostate biopsy: Five years of experience. Arch Ital Urol Androl. 2014; 86:340-3.

7. Gore JL, Shariat SF, Miles . et al. Optimal combinations of systematic sextant and laterally directed biopsies for the detection of prostate cancer. J Urol. 2001; 165:1554-9.

8. Martino P, Galosi AB, Bitelli M, et al. Imaging Working GroupSocieta Italiana Urologia (SIU); Società Italiana Ecografia Urologica Andrologica Nefrologica (SIEUN). Practical recommendations for performing ultrasound scanning in the urological and andrological fields. Arch Ital Urol Androl. 2014; 86:56-78.

9. Irani J, Fournier F, Bon D, et al. Patient tolerance of transrectal ultrasound-guided biopsy of the prostate. Br J Urol. 1997; 79:608-10.

10. Lee-Elliott CE, Dundas D, Patel U. Randomized trial of lidocaine vs lidocaine/bupivacaine periprostatic injection on longitudinal pain scores after prostate biopsy. J . 2004; 171:247.

11. Rabets JC, Jones JS, Patel AR et al. Bupivacaine provides rapid, effective periprostatic anaesthesia for transrectal prostate biopsy. BJU Int. 2004, 93:1216.

12. Damiano R, Cantiello F, Sacco, et al. Randomized placebocontrolled study of periprostatic local anaesthesic for transrectal ultrasound guided prostate biopsy. Arch Ital Urol Androl. 2004; 76:163.

13. Kaver I, Mabjeesh NJ, Matzkin H. Randomized prospective study of periprostatic localanesthesia during transrectal ultrasoundguided prostate biopsy. Urology. 2002; 59:405-8.
14. Rodriguez A, Kyriakou G, Leray E, et al. Prospective study comparing two methods of anaesthesia for prostate biopsies: apex periprostatic nerve block versus intrarectal lidocaine gel: review of the literature. Eur Urol. 2003; 44:195-200.

15. Kumar A, Griwan MS, Singh SK, et al. Is periprostatic nerve block a gold standard in case of transrectal ultrasound-guided prostate biopsy? Urol Ann. 2013; 5:152-6.

16. Giannarini $G$, Autorino R, Valent F. et al. Combination of perianal-intrarectal lidocaine-prilocaine cream and periprostatic nerve block for pain control during transrectal ultrasound guided prostate biopsy: a randomized, controlled trial. J Urol. 2009; 181:585-91.

17. Moussa AS, El-Shafei A, Diaz E, et al. Identification of the variables associated with pain during transrectal ultrasonography-guided prostate biopsy in the era of periprostatic nerve block: the role of transrectal probe configuration. BJU Int. 2013; 111:1281-6.

18. Rochester MA, Karena M, Brewster SF. A double-blind, randomized, controlled trial of topical glyceryl trinitrate for transrectal ultrasound guided prostate biopsy. J Urol. 2005; 173:418-420.

19. Koprulu S, Cevik I, Unlu N, et al. Size of the transrectal ultrasound probe makes no difference in pain perception during TRUS-Bx under adequate local anesthesia. Int Urol Nephrol. 2011; 44:29-33.

20. Dell'Atti L, Borea PA, Russo GR. Age: "a natural" in pain perception during the transrectal ultrasound-guided prostate biopsy procedure. Urologia. 2011; 78:257-61.

21. Philip J, McCabe JE, Dutta Roy S, et al. Site of local anaesthesia in transrectal ultrasonography-guided 12-core prostate biopsy: does it make a difference? BJU Int. 2006; 97:263-5.

22. Onur K, Gurdal I, Metin T, et al. Can Pain During Digital Rectal Examination Help us to Decide the Necessity and the Method of Anesthesia for Transrectal Ultrasound Guided Prostate Needle Biopsy? International Braz J Urol. 2007; 33:470-476.

\section{Correspondence}

Andrea Fabiani, MD (Corresponding Author)

andreadoc1@libero.it

Lucilla Servi, MD

lucilla.servi@sanita.marche.it

Fabrizio Fioretti, MD PhD

fa.fioretti@libero.it

Valentina Maurelli, MD

valentinamaurelli@hotmail.it

Gabriele Mammana, MD

gabriele.mammana@sanita.marche.it

Surgery Dpt., Section of Urology ASUR Marche Area Vasta 3

Macerata Hospital, Macerata, Italy

Alessandra Filosa, MD PhD

alessandrafilosa@yahoo.it

Section of Pathological Anatomy,

Department of Clinical Pathology, Area Vasta 3, ASUR Marche,

Macerata Hospital, Macerata, Italy

Flavia Tombolini, MD, Urology Resident

flavia.tombolini@gmail.com

Matteo Tallè, MD, Urology Resident

matteo.talle@gmail.com

Urologic Clinic, Polytechnic University of Marche Region 